\title{
Finite-difference time-domain model for elastic waves in the ground
}

\author{
Christoph T. Schroeder and Waymond R. Scott, Jr. \\ School of Electrical and Computer Engineering \\ Georgia Institute of Technology \\ Atlanta, GA 30332-0250 \\ USA
}

\begin{abstract}
A two-dimensional finite-difference model for elastic waves in the ground has been developed. The model uses the equation of motion and the stress-strain relation, from which a first order stress-velocity formulation is obtained. The resulting system of equations is discretized using centered finite-differences. A perfectly matched layer surrounds the discretized solution space and absorbs the outward traveling waves. The numerical model is validated by comparison to an analytical solution. The numerical model is then used to study the interaction of elastic waves with buried land mines. Results are presented for a buried antipersonnel mine. It can be seen, that an air-chamber within the mine is excited to resonant oscillations, which are clearly visible on the surface above the mine. The simulation results agree fairly well with experimental observations. Differences are mainly due to the numerical model being two-dimensional, whereas the experimental model is three-dimensional. Currently, the finite-difference model is being extended to three dimensions.
\end{abstract}

Keywords: land mine, elastic wave, acoustic, FDTD, finite-difference, numerical modeling

\section{INTRODUCTION}

A numerical finite-difference model for elastic waves in the ground has been developed and is described in this paper. The equation of motion and the stress-strain relation, together with a constitutive relation, form a set of first-order partial differential equations that completely describes the elastic wave motion in a medium. Introducing finite differences, this set of equations can be discretized and adapted to the finite-difference time-domain modeling scheme. Assuming that the field is known at one initial time $t_{0}$, this numerical scheme is used to determine the field values for all later times $t>t_{0}$.

The finite-difference model has been implemented in two dimensions. The solution space is discretized and a staggered finite-difference grid is introduced. The grid is surrounded by a perfectly matched layer, that absorbs the outgoing waves. The numerical model has been validated by comparison to an analytical solution. The analytical solution is obtained for elastic waves in a homogeneous half-space in form of an integral equation. Numerical integration must be used to determine the elastic waves. Excellent agreement is seen between the numerical model and the analytical solution.

The finite-difference model has been developed as part of a land mine detection project. In this project, a new technique is being investigated that synergistically uses both acoustic (elastic) and electromagnetic waves to detect land mines. ${ }^{1} \quad$ The numerical model has been very helpful for understanding the interaction of elastic waves with buried land mines.

The interaction of elastic waves with a buried antipersonnel mine has been explored. Two simple models for the TS-50 antipersonnel mine are investigated: one containing an air-filled chamber and one without an air-filled chamber. The interaction predicted by the simulation is in fairly good agreement with experimental results. ${ }^{2}$ The differences are mainly due to the numerical model being two-dimensional, while the experimental model is three-dimensional. A three-dimensional numerical model is currently under development.

Electronic Mail and Telephone: christoph.schroeder@ee.gatech.edu, 404-894-3123, waymond.scott@ee.gatech.edu, 404-894-3048 


\section{TWO-DIMENSIONAL NUMERICAL MODEL}

Elastic waves are launched into the earth and interact with a land mine that is buried in the ground. The interaction of the waves with the mine is to be investigated. For this problem, the earth can be modeled as an infinitely large half-space, bounded by a free surface. Under the conditions considered here, the earth can be approximated as isotropic and perfectly elastic and, thus, lossless.

\subsection{Finite-difference Model}

Figure 1 shows the two-dimensional finite-difference model. A normal point source is located on the free surface, exciting longitudinal (pressure) and transverse (shear) waves in the $x$ - $z$-plane. The wave fields are invariant in the $y$-direction. At $z=0$, a free surface bounds the solution space. A perfectly matched layer (PML) terminates the solution space at the remaining edges, absorbing all outward traveling waves. The space is discretized using a staggered finite-difference grid.

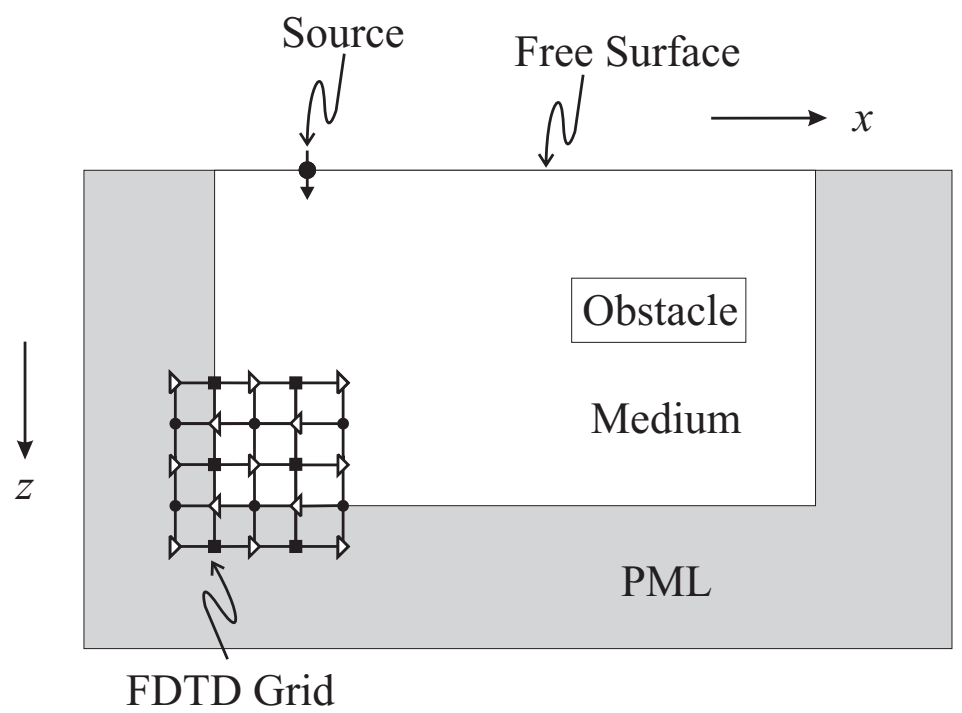

Figure 1. Two-dimensional finite-difference model.

A first-order velocity-stress formulation is chosen for the finite-difference model. ${ }^{3}$ Here, the equation of motion and the stress-strain relation are discretized, leading to a system of first order partial differential equations. Since only field components in the $x$ - $z$-plane are excited, the only non-zero field components are the three unknown stress components $\tau_{x x}, \tau_{x z}, \tau_{z z}$, and the two unknown particle velocities $v_{x}$ and $v_{z}$. Due to the invariance in the $y$-direction, all derivatives with respect to $y$ vanish. The wave motion is then completely described by a system of five partial differential equations:

$$
\begin{aligned}
\rho \frac{\partial v_{x}}{\partial t} & =\frac{\partial \tau_{x x}}{\partial x}+\frac{\partial \tau_{x z}}{\partial z} \\
\rho \frac{\partial v_{z}}{\partial t} & =\frac{\partial \tau_{x z}}{\partial x}+\frac{\partial \tau_{z z}}{\partial z} \\
\frac{\partial \tau_{x x}}{\partial t} & =(\lambda+2 \mu) \frac{\partial v_{x}}{\partial x}+\lambda \frac{\partial v_{z}}{\partial z} \\
\frac{\partial \tau_{z z}}{\partial t} & =(\lambda+2 \mu) \frac{\partial v_{z}}{\partial z}+\lambda \frac{\partial v_{x}}{\partial x} \\
\frac{\partial \tau_{x z}}{\partial t} & =\mu\left(\frac{\partial v_{x}}{\partial z}+\frac{\partial v_{z}}{\partial x}\right),
\end{aligned}
$$

where $\rho$ is the material density and $\lambda$ and $\mu$ are Lame's constants. These equations are discretized using centered finite-differences. Introducing the finite differences in space, $\Delta x$ and $\Delta z$, and in time, $\Delta t$, Eq. (1) and (3), for 
example, can be discretized as

$$
\begin{aligned}
\rho \frac{V_{x}^{k+0.5}(i-0.5, j-0.5)-V_{x}^{k-0.5}(i-0.5, j-0.5)}{\Delta t}= & \frac{T_{x x}^{k}(i, j-0.5)-T_{x x}^{k}(i-1, j-0.5)}{\Delta x} \\
& +\frac{T_{x z}^{k}(i-0.5, j)-T_{x z}^{k}(i-0.5, j-1)}{\Delta z}, \\
\frac{T_{x x}^{k+1}(i, j-0.5)-T_{x x}^{k}(i, j-0.5)}{\Delta t}= & (\lambda+2 \mu) \frac{V_{x}^{k+0.5}(i+0.5, j-0.5)-V_{x}^{k+0.5}(i-0.5, j-0.5)}{\Delta x} \\
& +\lambda \frac{V_{z}^{k+0.5}(i, j)-V_{z}^{k+0.5}(i, j-1)}{\Delta z} .
\end{aligned}
$$

Here, the capital letters mark the numerical value of the correspondent field component at a discrete location in space and time. For example, $V_{x}^{k+0.5}(i-0.5, j-0.5)$ stands for the numerical value of the particle velocity $v_{x}$ at $(x, z)=((i-0.5) \Delta x,(j-0.5) \Delta z)$ at time $t=(k+0.5) \Delta t$. Knowing $V_{x}^{k-0.5}, T_{x x}^{k}$ and $T_{x x}^{k}$, Eq. (6) can be solved for $V_{x}^{k+0.5}$, i.e. at the incremented time $t=(k+0.5) \Delta t$ :

$$
\begin{aligned}
V_{x}^{k+0.5}(i-0.5, j-0.5)= & V_{x}^{k-0.5}(i-0.5, j-0.5)+\frac{\Delta t}{\rho \Delta x}\left(T_{x x}^{k}(i, j-0.5)-T_{x x}^{k}(i-1, j-0.5)\right) \\
& +\frac{\Delta t}{\rho \Delta z}\left(T_{x z}^{k}(i-0.5, j)-T_{x z}^{k}(i-0.5, j-1)\right) .
\end{aligned}
$$

Similarly, $T_{x x}^{k+1}$ is obtained from Eq. (7):

$$
\begin{aligned}
T_{x x}^{k+1}(i, j-0.5)= & T_{x x}^{k}(i, j-0.5)+(\lambda+2 \mu) \frac{\Delta t}{\Delta x}\left(V_{x}^{k+0.5}(i+0.5, j-0.5)-V_{x}^{k+0.5}(i-0.5, j-0.5)\right) \\
& +\lambda \frac{\Delta t}{\Delta z}\left(V_{z}^{k+0.5}(i, j)-V_{z}^{k+0.5}(i, j-1)\right) .
\end{aligned}
$$

In the same manner, discretized equations can be obtained for all field components.

Figure 2 depicts the position of the field components in the finite-difference grid. Note that the velocity components and the stress components are not known at the same position in time and space, but offset by $\frac{\Delta t}{2}$ in time and by $\frac{\Delta x}{2}$ and $\frac{\Delta z}{2}$ in space. This leads to the introduction of the staggered grid and the so-called leapfrog algorithm. In the leapfrog algorithm, the field components are updated sequentially in time: the velocity components are calculated first, then the stress components from the velocity components, the velocity components again using the stress components and so on. Thus, knowing the field components throughout the entire space at the time $t_{0}$ and $t_{0}+0.5 \Delta t$, respectively, the field components can be determined for all later times $t>t_{0}$.

When implementing the finite-difference scheme, boundary conditions have to be treated in a special manner. Three different kinds of boundaries arise: the source point, internal boundaries (i.e. boundaries within the medium marked by a change in material properties), and external boundaries (i.e. the grid edges).

A normal point source is to be implemented on the free surface. This is done by forcing one field component to assume a specific time dependence. Here, the normal stress component $\tau_{z z}$ is excited by a differentiated Gaussian pulse.

The conditions at internal boundaries, i.e. at the interfaces between different media, are usually satisfied implicitly. However, to ensure numerical stability, the material properties have to be averaged for components on the boundary. While the material density $\rho$, appearing in the equation of motion, is averaged directly, the inverse of Lame's constants, $\lambda$ and $\mu$ from the stress-strain relation, must be averaged. For transitions in between similar materials, the averaging may be omitted. However, it is absolutely indispensable at an interface between media with greatly different material properties (for example, at an air-solid-interface).

Four external boundaries arise at the four outer grid edges. At its upper edge, the half-space is bounded by a free surface. Due to the continuity of normal stress, the normal stress components vanish at a free surface. In order to satisfy this condition, an extra row must be inserted into the finite-difference grid one step beyond the free-surface boundary.

In order to model the infinite half-space, all waves that are reaching the three remaining outer grid edges must be perfectly transmitted and absorbed. The boundary condition that does this most accurately is the Perfectly Matched 


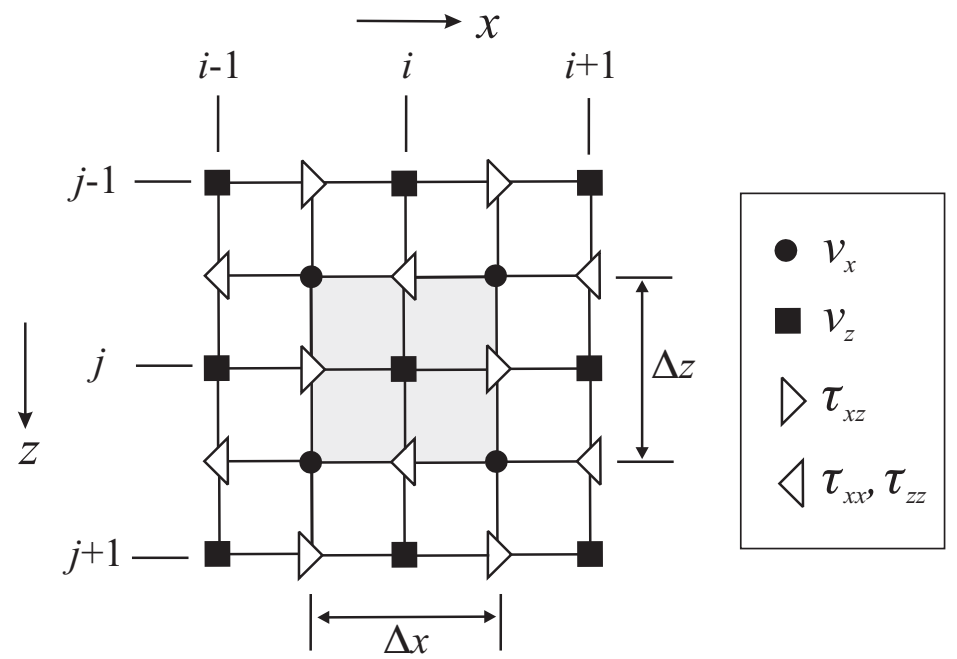

Figure 2. Part of the finite-difference grid. The field components are not known at the same locations, but offset by $\frac{\Delta x}{2}$ and $\frac{\Delta z}{2}$.

Layer (PML) boundary condition, first introduced by Berenger ${ }^{4}$ and adapted to elastodynamics by Chew and Liu. ${ }^{5}$ Here, a non-physical splitting of the wave fields allows the introduction of a lossy boundary layer that is perfectly matched to the solution space. It has been shown that an arbitrarily polarized wave incident on this PML medium is perfectly transmitted. The wave experiences the exact same phase velocity and wave impedance as in the solution space, while rapidly decaying along the axis normal to the PML-medium interface. The loss profile within the PML is chosen to be

$$
\sigma(i)=\sigma_{\max } \cdot\left(\frac{i-i_{\mathrm{PML}}}{N_{\mathrm{PML}}}\right)^{m},
$$

where $m \approx 2.1$ and $\sigma_{\max }=\frac{0.1 N_{\mathrm{PML}}}{\Delta t} ; N_{\mathrm{PML}}$ is the thickness of the PML in basis cells (here: $N_{\mathrm{PML}}=20$ ), and $i-i_{\mathrm{PML}}$ indicates the position within the PML. This loss profile (with a slightly different $\sigma_{\max }$ ) has been found to yield best performance in electromagnetic finite-difference modeling. ${ }^{6}$

\subsection{VALIDATION OF FINITE-DIFFERENCE RESULTS}

An analytic solution for the particle displacement fields in an infinite homogeneous half-space subjected to a normal harmonic line-load of finite width can be derived. ${ }^{7,8}$ Using a Fourier transform method, the particle displacement is obtained as an integral equation and can be determined using numerical integration.

The integral to be solved contains two poles and four branch cuts. Furthermore, the integration range goes from $-\infty$ to $\infty$. While the branch cuts do not impose a problem during numerical integration, the poles cannot be integrated numerically. To account correctly for the pole contributions, the poles must be subtracted from the integral and integrated analytically. To accelerate the integration, the infinite integration range may be truncated. However, for this to be possible, the integral has to converge sufficiently fast. In order to accelerate the convergence of the integral, an asymptotic approximation for the tails of the integrals is derived. This asymptotic approximation is subtracted from the integrals and integrated analytically.

To obtain the particle displacement in the time-domain, the displacement field must be Fourier transformed. Since the particle velocity is calculated in the FDTD simulation, the particle displacement is differentiated with respect to time, and the particle velocity is obtained.

\subsubsection{Comparison to FDTD Results}

In Fig. 3, the elastic waves due to a normal line load on a free surface are shown for both the analytic solution and the numerical simulation. A differentiated Gaussian pulse is launched at $t=0 \mathrm{~ms}$ from a source located at $(x, z)=(0,0) \mathrm{cm}$. The magnitude of the particle velocity fields is shown at $t=10 \mathrm{~ms}$. A pressure, a shear and a surface wave are seen to propagate. The pressure wave is the fastest of the three. The surface wave is propagating 
slightly slower than the shear wave and contains the most energy. A plane wave arises at the free surface, induced by the pressure wave. This wave is called a head wave. Two more head waves can be seen that, however, do not appear to originate from any other wave.

Figure 3(a) shows the analytical solution for the particle velocity field, and Fig. 3(b) shows the finite-difference result. The analytic and numerical result are seen to be indistinguishable. In Fig. 4, the particle velocity components of numerical and analytical result are plotted along the $x$-axis and along the $z$-axis. The agreement of numerical and analytical results is very good. It can be seen that the finite-difference model predicts waves that travel slightly slower than those of the analytical solution. This is due to numerical dispersion within the finite-difference grid. By choosing a smaller grid spacing the effect of numerical dispersion can be reduced. The velocity component $v_{x}$ must be zero along the $z$-axis at $x=0 \mathrm{~cm}$. For the analytical solution, however, the $v_{x}$ component is seen to have a nonzero value along the $z$ axis, whereas it is seen to be zero for the finite-difference solution. This is due to an error introduced from an interpolation algorithm used for the analytical solution.

\section{INTERACTION OF ELASTIC WAVES WITH THE TS-50 ANTIPERSONNEL MINE}

The interaction of elastic waves with a TS-50 antipersonnel mine, buried in sand, is to be investigated. Two simple models for the TS-50 mine are shown in Fig. 5. In the first model, a small chamber filled with air is located on top of the mine's main chamber containing plastic explosives. The second model does not contain an air-filled chamber. The elastic properties of the materials used for the numerical simulation are summarized in Table 1.

Table 1. Parameters used for finite-difference simulation.

\begin{tabular}{|clll|}
\hline Sand & Shear wave velocity & $c_{s, \text { sand }}$ & $87 \mathrm{~m} / \mathrm{s}$ \\
& Pressure wave velocity & $c_{p, \text { sand }}$ & $250 \mathrm{~m} / \mathrm{s}$ \\
& Material density & $\rho_{\text {sand }}$ & $1400 \mathrm{~kg} / \mathrm{m}^{3}$ \\
\hline \multirow{2}{*}{ Mine } & Shear wave velocity & $c_{s, \text { mine }}$ & $1100 \mathrm{~m} / \mathrm{s}$ \\
& Pressure wave velocity & $c_{p, \text { mine }}$ & $2700 \mathrm{~m} / \mathrm{s}$ \\
& Material density & $\rho_{\text {mine }}$ & $1200 \mathrm{~kg} / \mathrm{m}^{3}$ \\
\hline \multirow{2}{*}{ Air } & Shear wave velocity & $c_{s, \text { air }}$ & $0 \mathrm{~m} / \mathrm{s}$ \\
& Pressure wave velocity & $c_{p, \text { air }}$ & $330 \mathrm{~m} / \mathrm{s}$ \\
& Material density & $\rho_{\text {air }}$ & $1.3 \mathrm{~kg} / \mathrm{m}^{3}$ \\
\hline
\end{tabular}

Figure 6 shows the excitation as a function of time. The excitation is a differentiated Gaussian pulse with a center frequency of $800 \mathrm{~Hz}$ and launched at $t=0 \mathrm{~ms}$. The source is located on the free surface at $x=0 \mathrm{~cm}$. The left edge of the mine lies at $x=45 \mathrm{~cm}$. Its upper edge is $2 \mathrm{~cm}$ underneath the surface of the ground.

Fig. 7 and 8 show the interaction of the excited waves with the antipersonnel mine. The mine in Fig. 7 contains an air-filled chamber, while the mine in Fig. 8 does not. The magnitude of the particle velocity is plotted on a logarithmic scale. The particle velocity field is shown at four different times: at $t=2 \mathrm{~ms}, t=5 \mathrm{~ms}, t=6 \mathrm{~ms}$ and $t=10 \mathrm{~ms}$.

At $t=2 \mathrm{~ms}$, a pressure, a shear and a surface wave have been launched. The pressure wave, the fastest of the three, just hits the mine. For the mine with the air-filled chamber, some energy is seen to be trapped in between the surface and the mine, while for the mine without the air-filled chamber no strong interaction occurs. At $t=5$ ms and $t=6 \mathrm{~ms}$, the surface wave hits the mine and is partially transmitted and partially reflected. At $t=10 \mathrm{~ms}$, the waves reflected from the mine are clearly seen. For the mine without the air-filled chamber, the reflected waves are only weakly dispersed. However, for the case with the air-filled chamber, the reflected waves are seen to be strongly dispersed. Energy is still trapped in between the mine and the surface, though the incident surface wave has already passed by.

In Fig. 9, a waterfall graph of the particle velocity $v_{y}$ at the surface is shown for a mine with and without the air-filled chamber. Here, $v_{y}$ is plotted as a function of time and vertically offset by the distance from the source. 


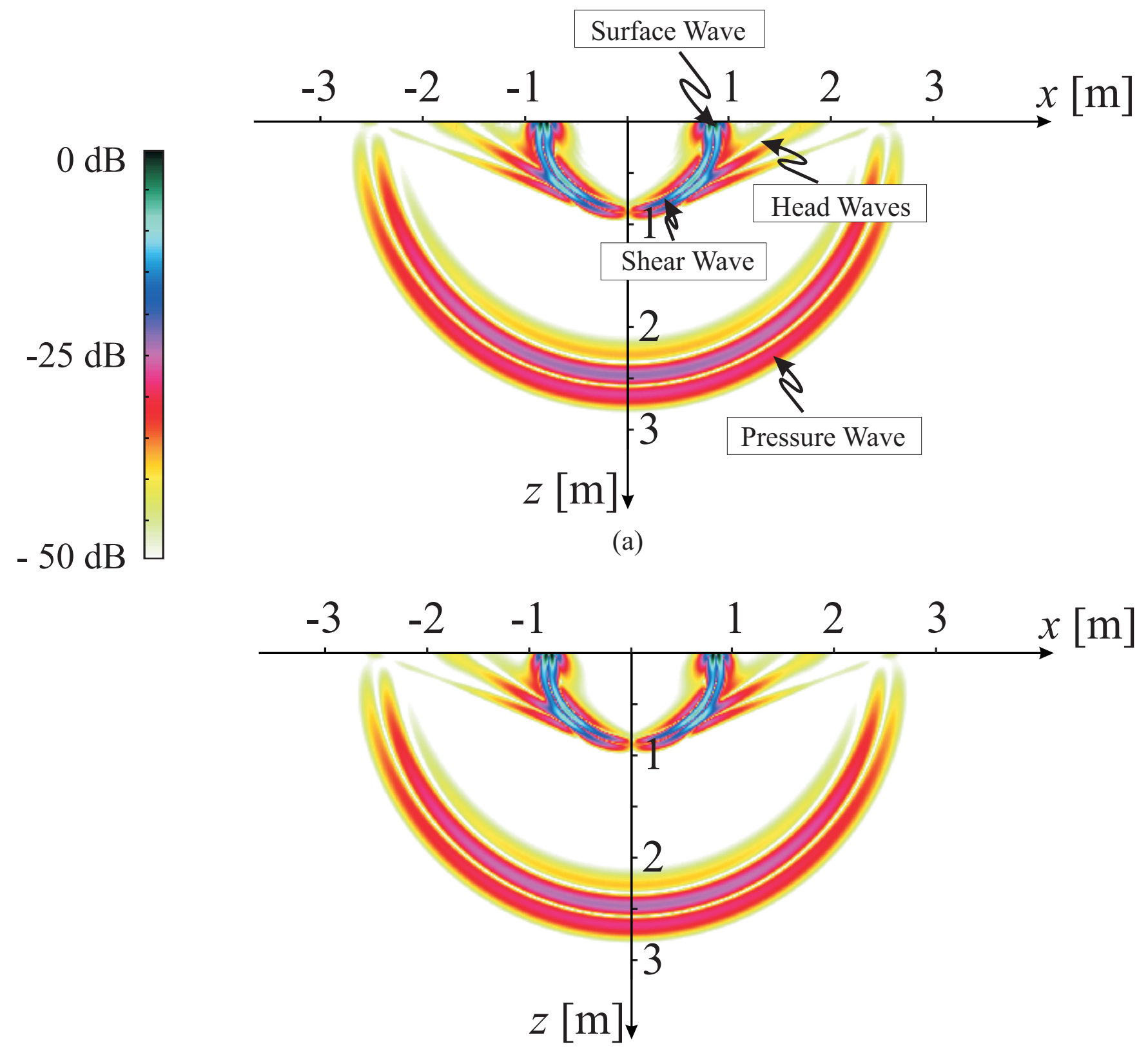

(b)

Figure 3. Plot of the magnitude of the elastic waves in a half-space subjected to a normal line-load at $t=10 \mathrm{~ms}$ : (a) analytical result; (b) finite-difference result.

The slope of the traveling waves in the graph indicates the wave speed. Thus, by looking at their slope, the different waves can be distinguished. A surface wave $(\mathrm{S})$ and a pressure wave $(\mathrm{P})$ are seen to be incident onto the mine. The pressure wave is reflected and transmitted by the mine. It converts into a reflected pressure wave (not visible in the graph), a reflected surface wave (rSP), a transmitted pressure wave $(\mathrm{P})$ and a transmitted surface wave (SP). These waves are weak, due to the limited energy content of the pressure wave. The pressure wave is seen to travel faster over the mine than in the sand. This is due to the higher wave speed in the mine. The incident surface wave gives rise to a reflected pressure wave (rPS), a reflected surface wave (rSS), a transmitted pressure wave (PS) and a transmitted surface wave (S). For the mine with the air-filled chamber (Fig. 9(a)), a strong resonance can be seen at 


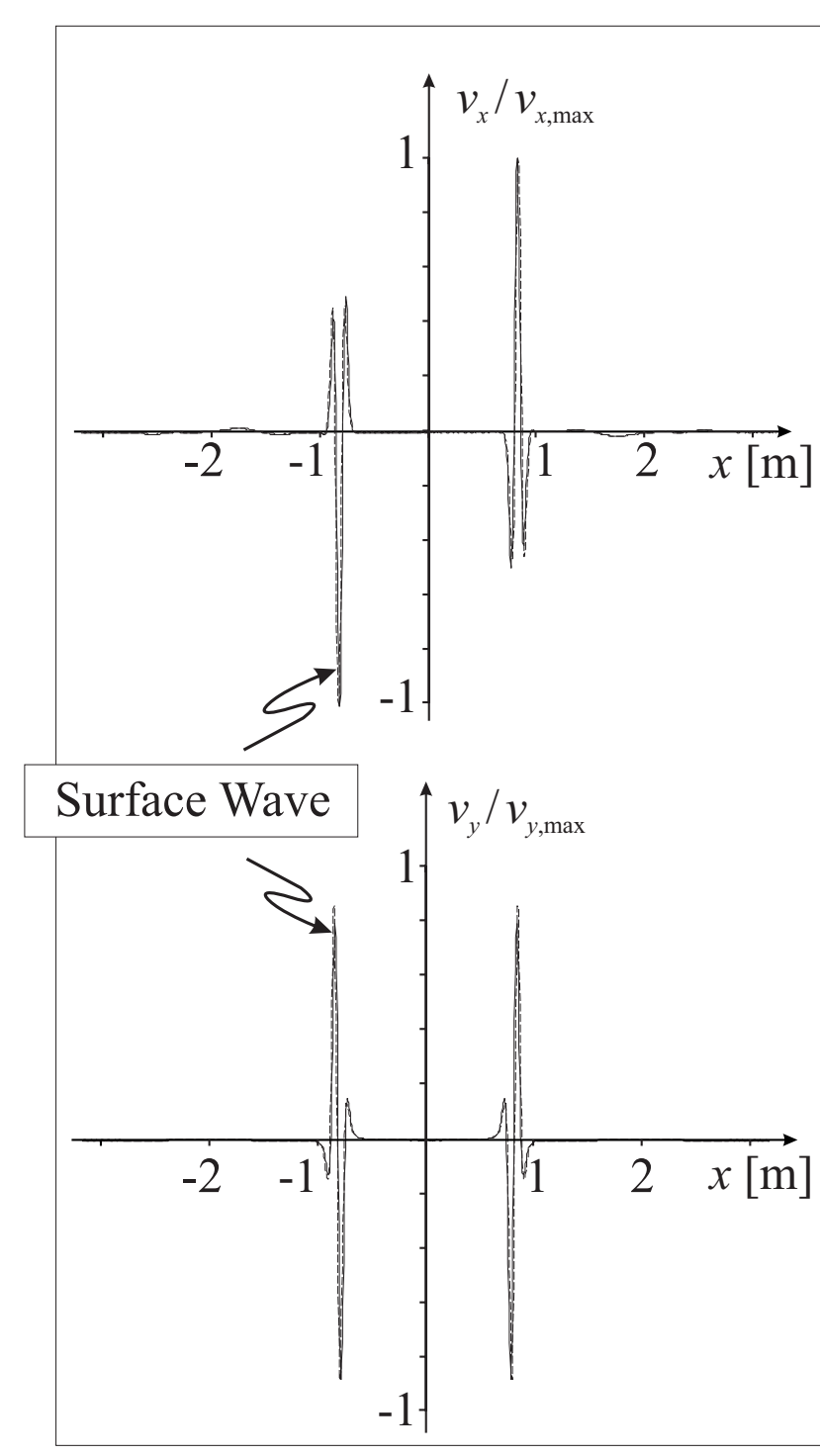

(a)
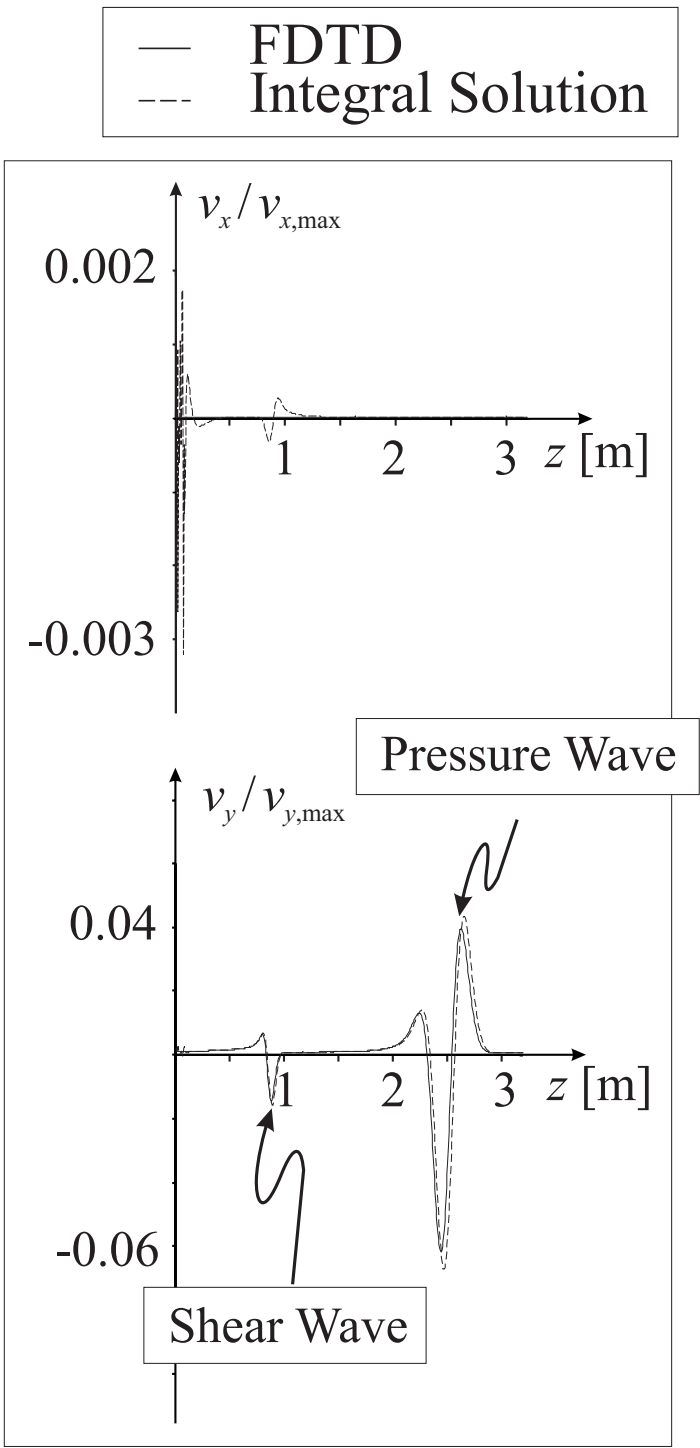

(b)

Figure 4. Comparison of Analytic and FDTD results: Displacement fields (a) along the $x$-axis, (b) along the positive $z$-axis. The particle velocity $v_{x}$ along the $y$-axis is nonzero for the integral solution, due to an interpolation algorithm used during the Fourier transform.

the mine location. The resonance remains at the mine, even after the incident surface wave has passed by, and causes the surface wave to disperse. This resonance is due to the trapped energy in between the mine and the surface. For the mine without the air-filled chamber (Fig. 9(b)), no resonance occurs and the wave dispersion is weak.

The results obtained from the simulation are in fairly good agreement with experimental results. ${ }^{2}$ A similar resonance for the mine with the air-filled chamber is observed in the experimental model. In the experimental model, the resonance is stronger than in the numerical model, whereas in the numerical model the reflections from the mine are much stronger. These differences are due to the numerical model being two-dimensional, whereas the experimental model is three-dimensional. 


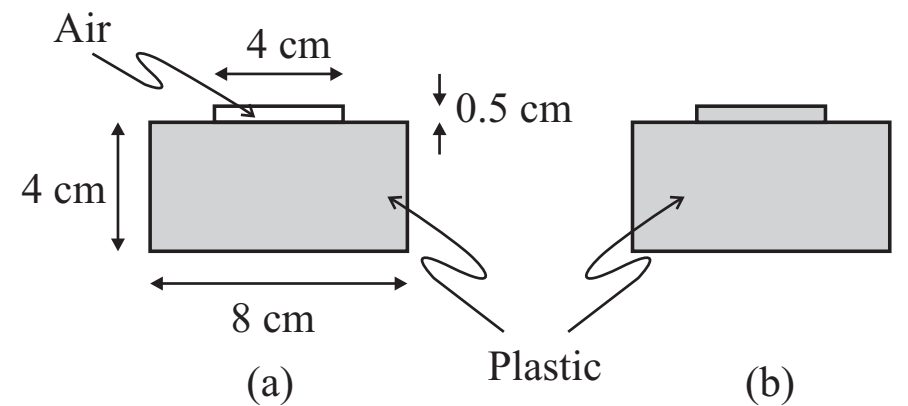

Figure 5. Simple model of the antipersonnel mine TS-50 (a) with and (b) without an air-filled chamber.

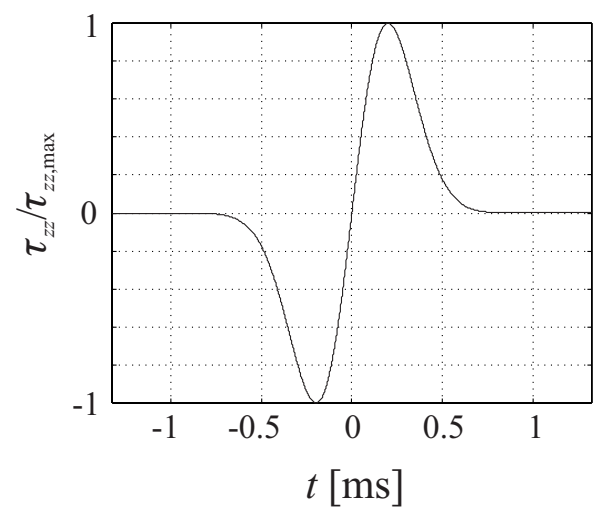

Figure 6. Source excitation: differentiated Gaussian pulse with a center frequency $800 \mathrm{~Hz}$.

\section{THREE-DIMENSIONAL NUMERICAL MODEL}

The numerical finite-difference model is currently being extended to three-dimensions. A preliminary result is shown in Fig. 10. The particle velocity fields are plotted on the surfaces of a cube. The upper surface represents the surface of the ground and is modeled as a free surface. The cube faces on the left and on the right represent cross sections of the ground. A normal point source is located at $(x, y, z)=(0,0,0) \mathrm{cm}$. A differentiated Gaussian pulse is launched. Surface, shear and pressure waves are excited. As in the two-dimensional simulation, head waves arise at the free surface. On the cross sections, propagating surface, shear, pressure waves and head waves are visible, whereas on the surface only the surface waves and the head waves can be seen.

\section{CONCLUSIONS}

A finite-difference model has been developed and implemented in two dimensions. The model has been verified by comparison to an analytical solution. The finite difference model has been used to investigate the interaction of elastic waves with a buried antipersonnel mine. Results are obtained for a TS-50 antipersonnel mine with and without an air-filled chamber. For both cases, strong reflections are observed. For the mine with the air-filled chamber, a strong resonance at the mine location occurs. This resonance is caused by energy being trapped in between the mine and the surface. The results from the numerical model are found to be in fairly good agreement with experimental results. A three-dimensional model is currently being developed and will give further insight into the wave-mine interaction within the ground.

\section{ACKNOWLEDGMENTS}

This work is supported in part under the OSD MURI program by the US Army Research Office under contract DAAH04-96-1-0048. 


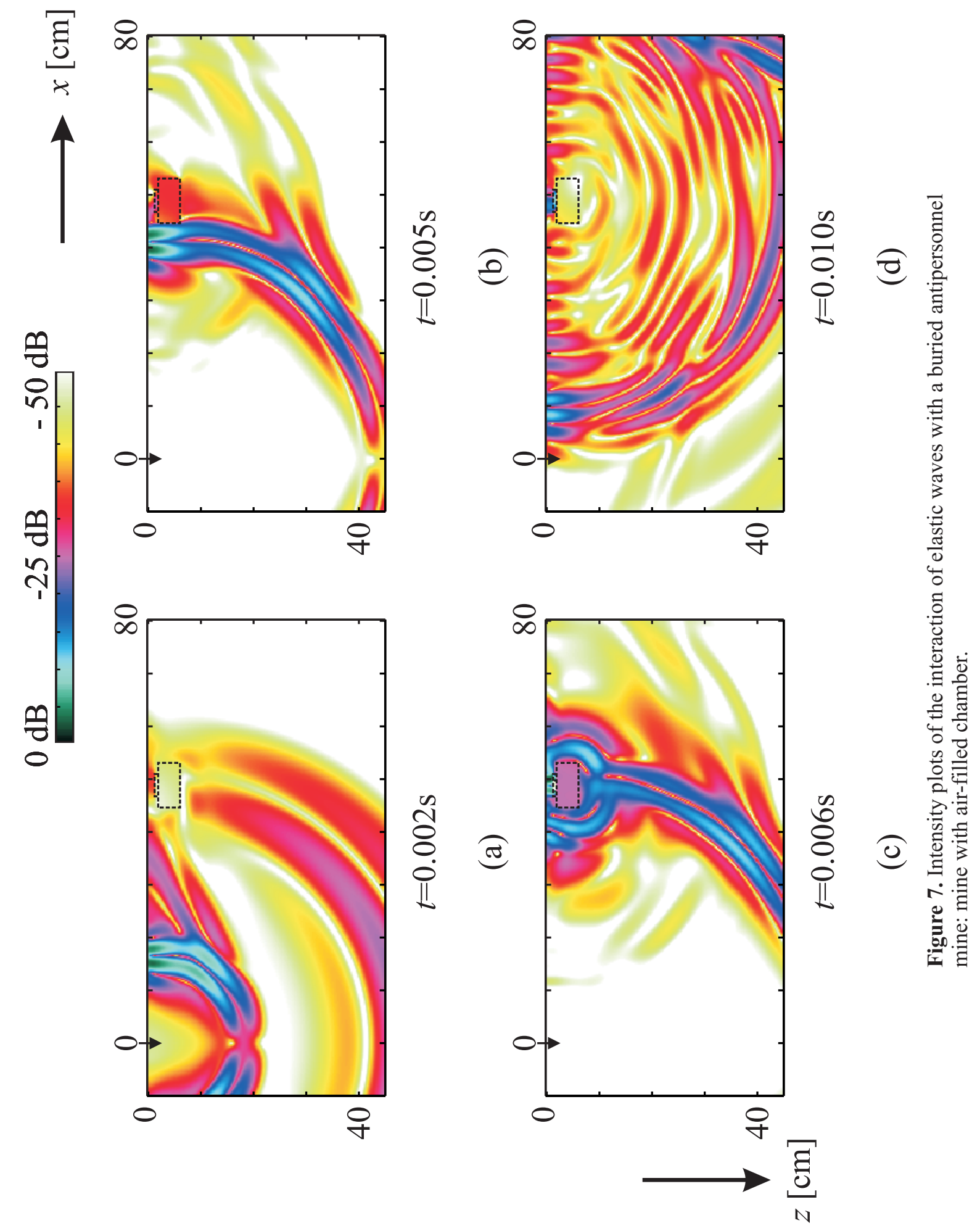

Figure 7. 


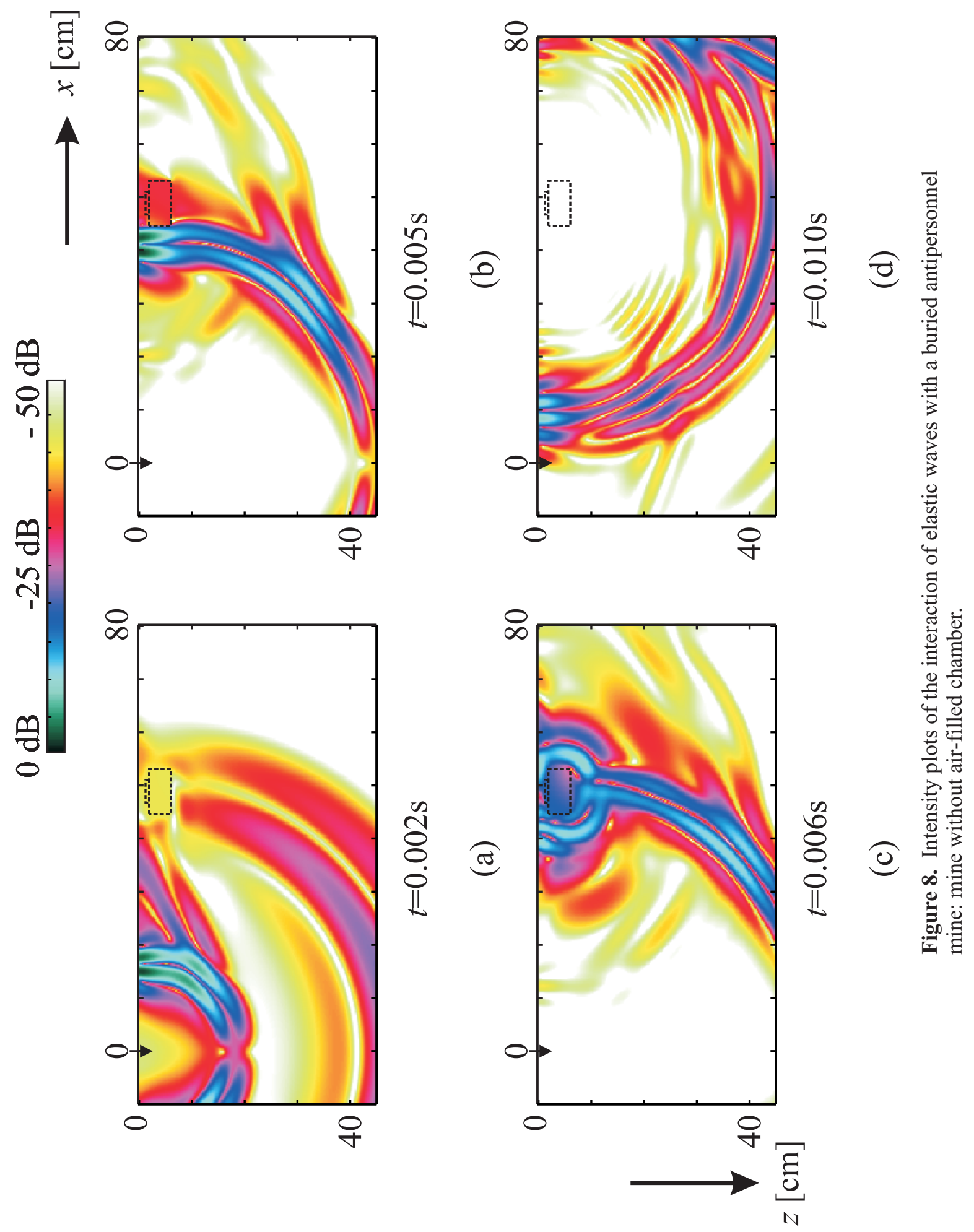

Figure 8 . 


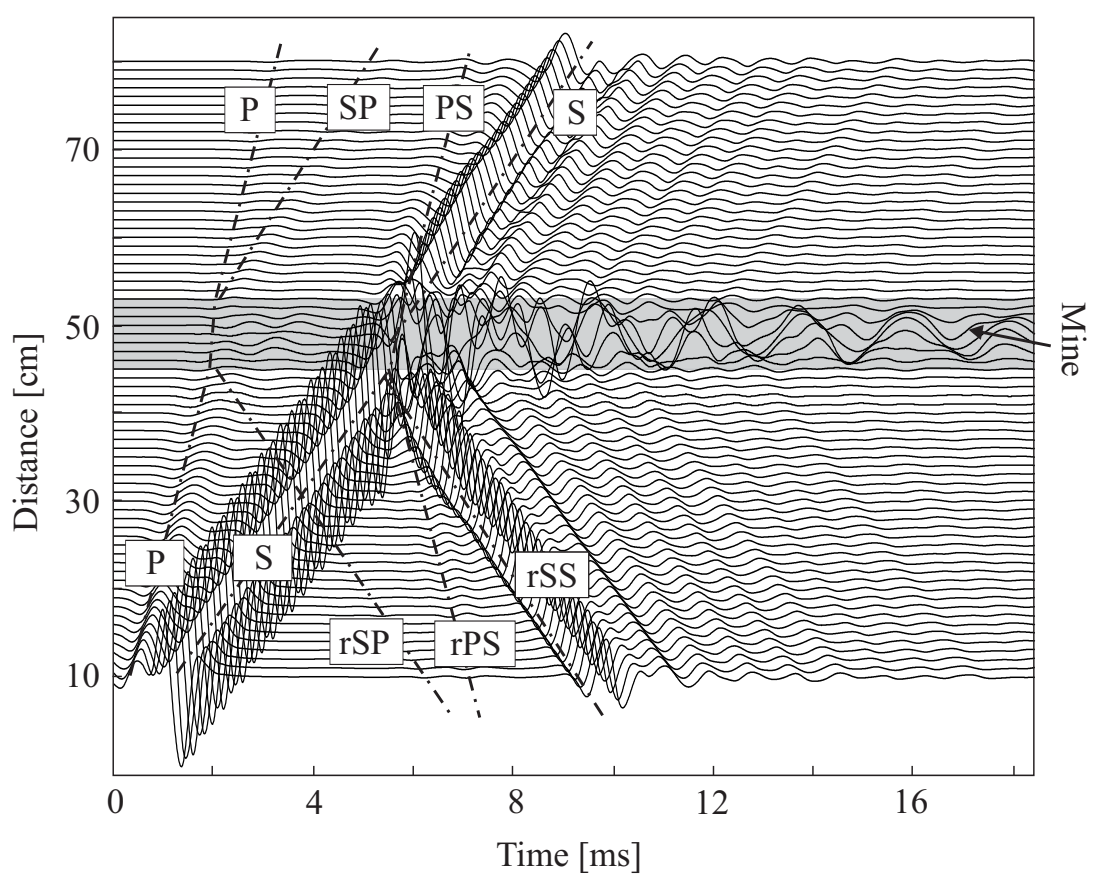

(a)

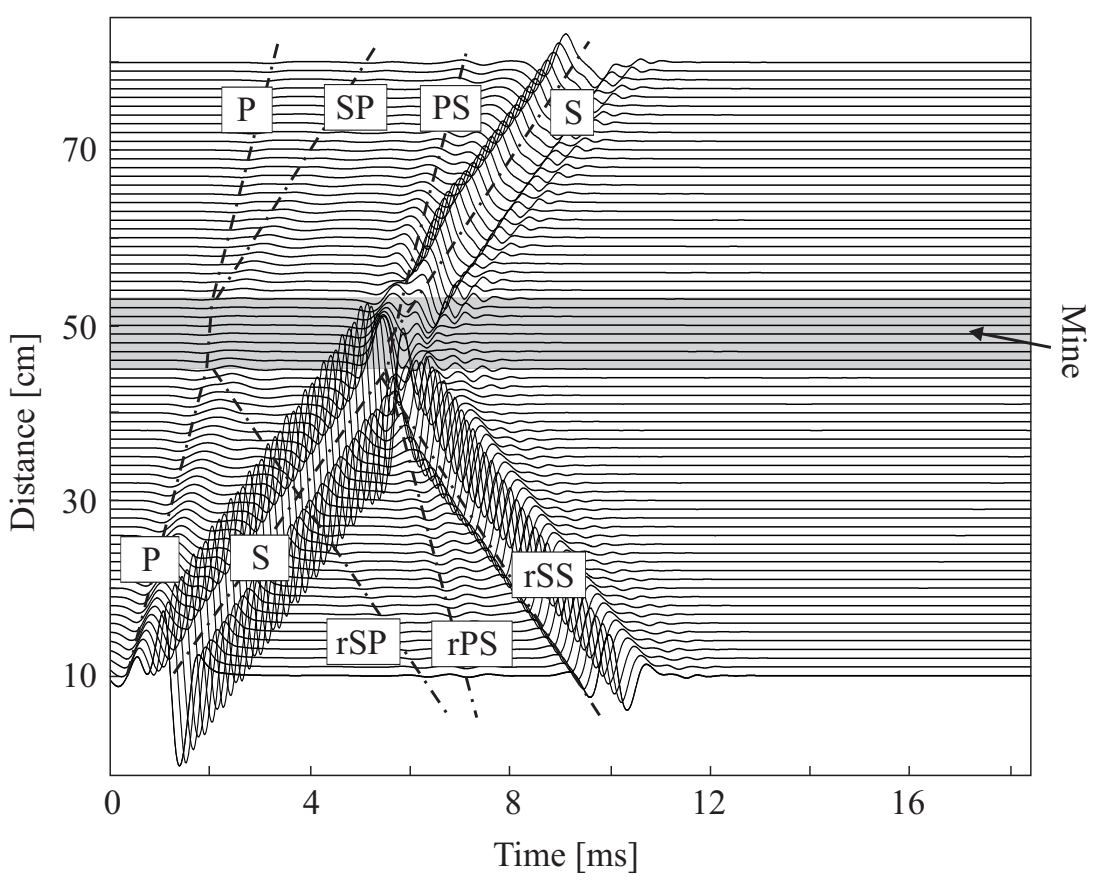

(b)

Figure 9. Interaction of elastic waves with a buried antipersonnel mine TS-50; waterfall graph of the particle velocity $v_{y}$ at the surface for (a) a mine with the air-filled chamber and (b) a mine without the air-filled chamber. For the mine with the air-filled chamber, resonant vibrations can be seen at the mine location, which are due to energy being trapped in between the mine and the surface. P: pressure wave; S: surface wave; SP: surface wave due to pressure wave; PS: pressure wave due to surface wave; rSP: reflected surface wave due to pressure wave; rPS: reflected pressure wave due to surface wave; rSS: reflected surface wave due to surface wave. The vertical axis indicates the distance from the source. 


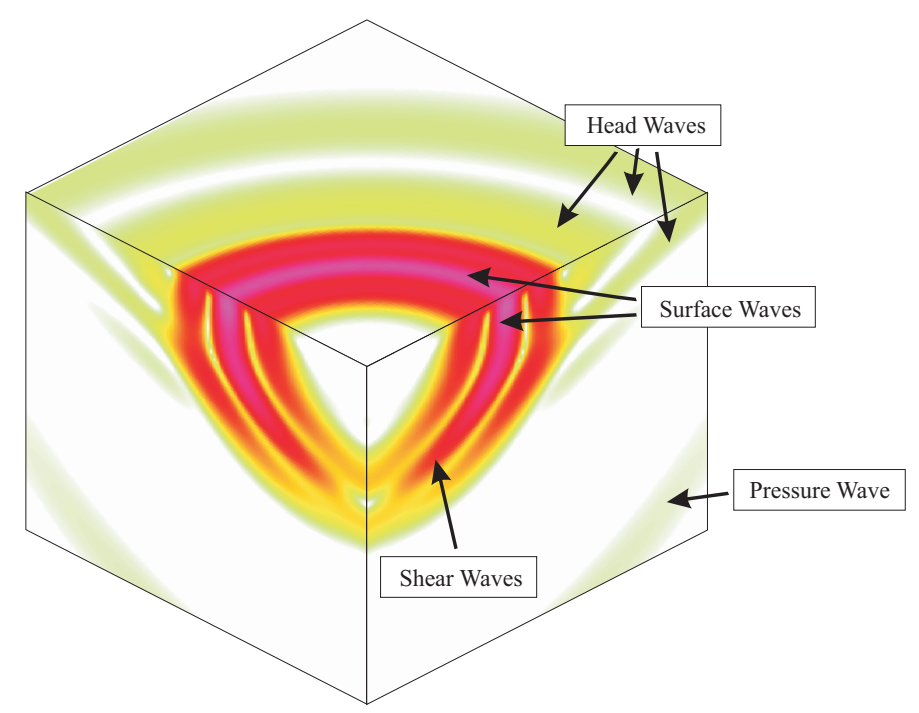

Figure 10. Propagating elastic waves in a three-dimensional half-space.

\section{REFERENCES}

1. W. R. Scott, Jr., C. T. Schroeder, and J. S. Martin, "An acousto-electromagnetic sensor for locating land mines," in Detection and Remediation Technologies for Mines and Minelike Targets III, Proc. SPIE 3392, pp. 176-186, 1998.

2. W. R. Scott, Jr. and J. S. Martin, "Experimental investigation of the acousto-electromagnetic sensor for locating land mines," in Detection and Remediation Technologies for Mines and Minelike Targets IV, Proc. SPIE , 1999.

3. J. Virieux, "P-SV wave propagation in heterogeneous media: Velocity-stress finite-difference method," Geophysics 51, pp. 889-901, 1986.

4. J.-P. Berenger, "A perfectly matched layer for the absorption of electromagnetic waves," J. Comput. Physics 114, pp. 185-200, 1994.

5. W. C. Chew and Q. H. Liu, "Perfectly matched layer for elastodynamics; a new absorbing boundary condition," J. Comput. Acoustics 4, pp. 341-359, 1996.

6. T. W. Hertel, Pulse Radiation from an Insulated Antenna: An Analogue of Cherenkov Radiation from a moving Charge, Master's Thesis, Georgia Institute of Technology, 1998.

7. G. F. Miller and H. Pursey, "The field and radiation impedance of mechanical radiators on the free surface of a semi-infinite isotropic solid," Proc. R. Soc. A 223, pp. 521-541, 1954.

8. K. F. Graff, Wave Motion in Elastic Solids, Dover Publications, Inc., 1975. 


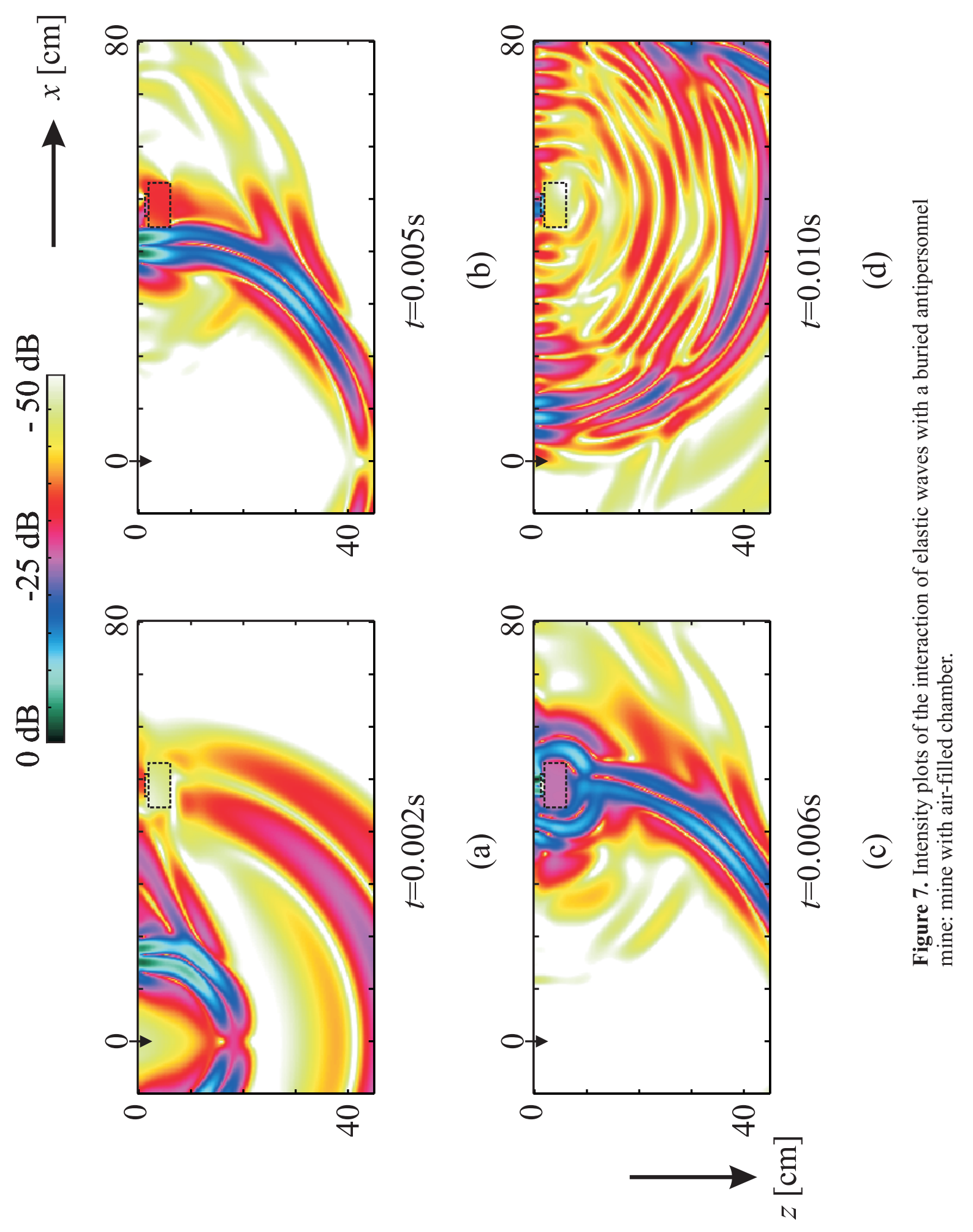




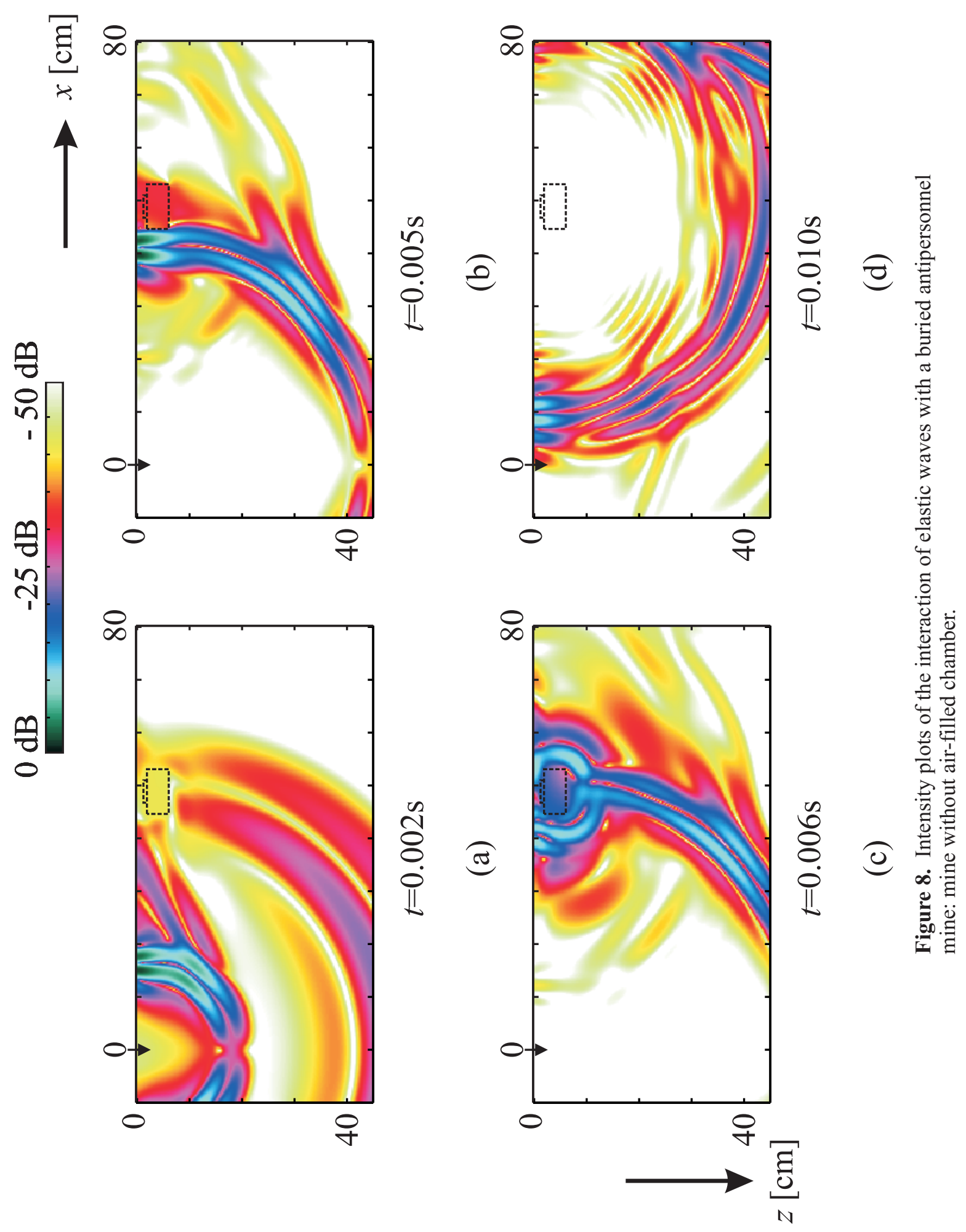

